\title{
A one-way, static Markov model estimating the social and economic burden of chronic obstructive pulmonary disease in Bulgaria for patients who smoke or don't.
}

\author{
Konstantin Tachkov*, Konstantin Mitov, Alexandra Savova, Maria Kamusheva \\ Medicinski Universitet Sofia, Sofia, Bulgaria
}

\begin{abstract}
Aims: We aimed at estimating the societal and economic burden of COPD in Bulgaria for the current prevalence and compare local to European trends.

Materials and methods: Between 2015-2016 data on 426 patients was collected in an ambispective, representative national study for Bulgaria. We recorded the distribution of patients as per GOLD severity classification and calculated the costs of treatment. A one-way Markov model was constructed for the cohort, examining effectiveness of smoking cessation, mortality and value of life years saved. The model was transposed to the country's entire COPD population $(447,070)$ according to smoking status to evaluate the future economic burden and quality of life.

Results: Cost of therapy increased as disease progressed (438€ for GOLD A to 790€ for GOLD D ( \pm 85.56)). 10-year mortality was higher for smokers $(54.9 \%$ vs. $49.7 \%)$. The cohort model revealed lower costs and QALYs for smokers vs. non-smokers (1,520,560.4 BGN; 1786.8 vs. 1,866,675.18; 2146.9). Incremental ratios were 954.1 per QALY gained and 6530.46 per life year saved. The population estimates were approximately 1.1 billion BGN and 1,280,308 QALYs for smokers vs. 556 million BGN and 640,887 QALYs for non-smokers, with ICERs of 860.32 BGN per QALY gained and 8,441.82 per life year saved.

Conclusion: Non-smokers experience higher costs and higher quality of life. The majority of COPD patients in the general population smoke $(310,465)$, accounting for nearly twice the cost of therapy than non-smokers $(\mathbf{1 3 6 , 6 0 5})$, who live longer with better QoL. Smoking cessation campaigns could help lessen the health authority's economic burden.
\end{abstract}

Keywords: Markov model, Quality of life, Cost-effectiveness, Economic burden, Societal burden.

Accepted on September 5, 2018

\section{Introduction}

Due to their late onset, long duration and higher comorbidities, chronic diseases have regularly been regarded as diseases with a high social and economic burden. Ever since the first report published by the World Bank in 1990 on their global burden [1], chronic conditions have been monitored both nationally and internationally with the aim of better education and understanding of their complex nature, as well as a helping tool for decision makers to estimate future costs.

Chronic respiratory disorders are among the most widely distributed non-communicable diseases, with prevalence rates in Europe of approximately 30 million people over the age of 45 [2]. Worldwide, Chronic Obstructive Pulmonary Disease (COPD) accounts for 4 million deaths annually and the World Health Organization (WHO) estimates that by 2030 it would be the second most common diagnosis, after diabetes and by 2020 it will be the fourth leading cause of death [3]. Estimating the disease prevalence rate has proven difficult, due to the high overlap between COPD and asthma and the many risk factors associated with developing COPD, such as tobacco smoke, air pollution, occupational hazards and others [4,5]. Many countries present a prevalence rate of $6 \%$ [6] in contrast to the established global rates of $11.8 \%$ for men and $8.5 \%$ for women [7].

In Bulgaria no official records on COPD prevalence exists, although there is an official report by the Ministry of Health that estimates the prevalence of risk exposure such as tobacco smoking and air pollution in the country. Approximately $39 \%$ of the population over 40 smokes (1.55 million people out of 3.98 million) and expert opinions in the country put the number of people with COPD at around 450 thousand. This estimate is in accordance with global literature regarding COPD development, which state that approximately $20 \%$ of smokers and $11 \%$ of non-smokers develop the condition $[8,9]$.

Markov models are an important tool in the repertoire of health analysts for projecting future burdens when lots of uncertainties are associated with a particular disease. Currently most information and prognoses are performed in high-income 
countries such as the Netherlands, where Feenstra et al. have developed a dynamic, multi-aspect lifetime model [10], or in the United Kingdom, where Atsou et al. have used modelling to investigate the effectiveness of smoking cessation programmes [11]. For a country with limited healthcare resources and high smoking rates as Bulgaria, projecting the future burden of chronic diseases, especially COPD is of great importance in order to adequately distribute resources. This study aims to help fill this gap in knowledge and give information on a developing region within the eastern part of the European Union.

The goal of the study is to build a one-way static Markov model for smokers and non-smokers with COPD in Bulgaria and to explore the cost-effectiveness of their therapy.

The point of view is that of the reimbursement system and time horizon is 10 years.

\section{Materials and Methods}

\section{Design of data collection}

During the period of 2015-2016 a national ambispective, follow-up study was conducted within 19 regions of the country. 426 patients were collected on a random principleevery $5^{\text {th }}$ candidate who entered pneumonologist's practice was asked to participate, and written consent was obtained. Bulgarian Science Fund approved the study. Data collected included pharmacotherapy, smoking status, age, sex, FEV1/ FEC, occupation, and disease stage.

\section{Cost and utility data}

Further analysis of the costs of therapy per disease stage was performed. Prices of medicines were obtained from the webpage of the National Council of Pricing and reimbursement and yearly costs per patient were calculated by multiplying the prescribed quantities of medicines with respective prices [12] (Table 1).

Utilities for every severity stage were obtained from Chandra et al. [13]. Four stages according to GOLD 2015 classification were used-GOLD A, GOLD B, GOLD C, GOLD D [14].

\section{Structure of the model}

We constructed a one-way Markov model, using quality of life data and transitional probabilities from available literature $[13,15]$. The model consisted of 5 states- 4 for each disease stage (GOLD A-D) and one absorbing state (Death), and estimated the associated costs and quality of life for a 10 -year period (Figure 1). Two models were constructed-one for the cohort and one for the general population, which estimated the economic and social burden for smokers and non-smokers. The initial distribution of patients per disease stage in our cohort(7.04\% GOLD A, 36.5\% GOLD B, 15.3\% GOLD C, and $41.3 \%$ GOLD D)-was used as a basis for the distribution in the general population.

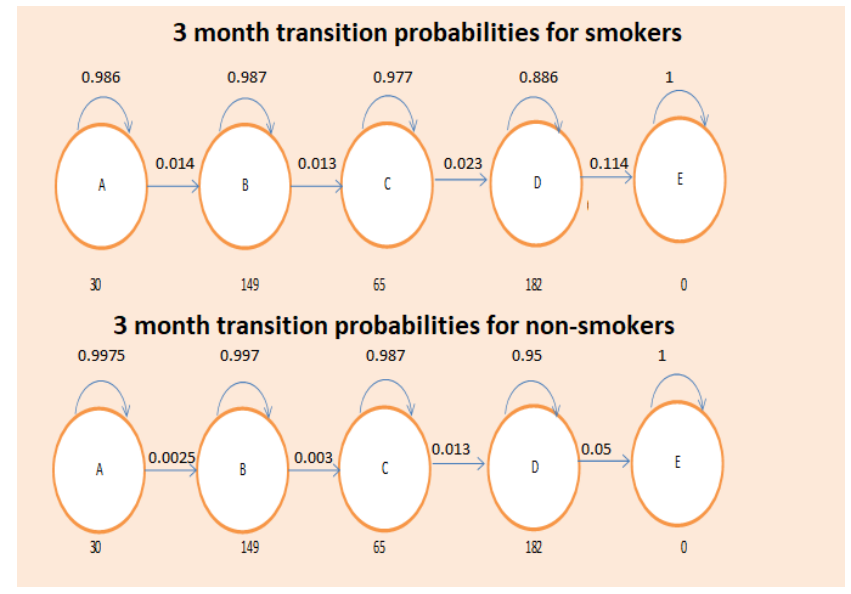

Figure 1. Structure of the Markov model with estimated 3-month transition probabilities.

The incremental cost-effectiveness ratio (ICER) was calculated for the two risks groups with the following assumptions: All 426 patients were smokers, or all 426 patients were nonsmokers or ex-smokers, since lung function rapidly improves within a year of quitting smoking [16]. The constructed model was then applied with the prevalence estimates, which were calculated for the country, assessing the future burden on the National Health Insurance Fund (NHIF).

Mortality was calculated for both groups of patients, and the value of each Life Year Saved at the end of the 10-year period was estimated.

\section{Sensitivity analysis}

To test the robustness of the results, deterministic and probabilistic sensitivity analyses (Tornado Diagram and Monte Carlo simulation) were conducted and the corresponding costeffectiveness acceptability curve (CEAC) was constructed only for the cohort model. For the deterministic analysis the cost and utilities were varied within a $\pm 30 \%$ interval and ICERs were recalculated. For the probabilistic sensitivity analysis 1000 iterations of the calculations were performed, both in the cohort and population model, using a random sampling of the values within the $95 \%$ CI. The cost-effectiveness acceptability curve analysed the minimum and maximum points, at which none of the point values were within the price scope, and at which all values were $100 \%$ within the scope.

\section{Results}

The most well-established risk factor that leads to disease progression-tobacco smoking, affected our population as well and our previous analysis showed that smokers have approximately 4.5 times higher odds of developing the disease than non-smokers. This observation was more pronounced in male smokers.

One of the more prevalent trends observed was the late diagnosis of the disease within our cohort. GOLD D and B were among the most populated groups (41.3\% and $36.5 \%)$, 
whereas only $7.02 \%$ of all patients were classified as GOLD A, which affected the corresponding therapy costs and quality of life (Table 2). Another parameter which was severely affected by the distribution of patients was the mortality. Late stage patients are the group with the highest rates of exacerbations and mortality due to disease. This in turn influenced the progression of the patients and the high death rates within the Markov model (Table 3).

The model predicted that the best disease control was accomplished in the non-smoker cohort in the early stages of the disease. At the end of the 10-year period, 176 non-smokers had died, with the majority of them coming from the proportion with GOLD D classification (41.3\% mortality rate). Only 3 patients had progressed from GOLD A, and both GOLD D and C patients showed better disease control than the smoking population (135 in GOLD B, and 52 in GOLD C at year 10). In contrast, smokers exhibited a higher turnover rate throughout the model, progressing quickly with a higher mortality rate. At year 10,53.7\% of the population had died, according to the model, with 17 patients left in GOLD A, 135 in GOLD B, 52 in GOLD C, and 36 in GOLD D. As a result, non-smokers accumulated more costs, but also better quality of life than smokers, with an incremental ratio of 954.1 BGN for each QALY gained. The incremental value of each life year saved (LYS) was estimated to be 6530.46 BGN.

Using the same matrix for the entire Bulgarian population revealed similar mortality rates $(54.9 \%$ for smokers $v s .42 .7 \%$ for non-smokers), but higher costs for the smoking population, due to significantly larger proportion of smokers in the country (Table 4). The accumulated costs for the smoking population were approximately 1.1 billion BGN, with 1.24 million QALYs, while for the non-smokers the costs and QALYs were 558 million BGN and 677.5 thousand respectively. The incremental ratios for each QALY gained were 860.32, and 8441.82 BGN for each life year saved. Effective disease and progression control within the population is more valuable, leading to bigger savings for the NHIF.

\section{Sensitivity analysis}

Both types of sensitivity analyses utilized revealed that the current therapy is cost-effective for both groups of patients and homogeneity of results (Figures 2 and 3). Increasing the costs of therapy for non-smokers by $30 \%$ led to an incremental ratio of $+2516.29 \mathrm{BGN}$, while reducing the QALYs by $30 \%$ led to a negative ICER of -2557.40. Increasing the costs for nonsmokers reduced the ICER for each LYS to 2516.29, while the ICER fell to -5768.58 BGN when reducing the LYS by $30 \%$. For both models the estimated incremental ratios were costeffective, since all were beneath the threshold of 3 times GDP per capita. The probabilistic Monte Carlo simulation of the results showed a homogenous distribution of values around the means -954.1 BGN and 860.32 BGN for the cohort and population models, respectively. The corresponding costeffectiveness acceptability curves were from 900 to 1050 BGN for the cohort model, and from 800 to 930 BGN for the population model (Figure 4).

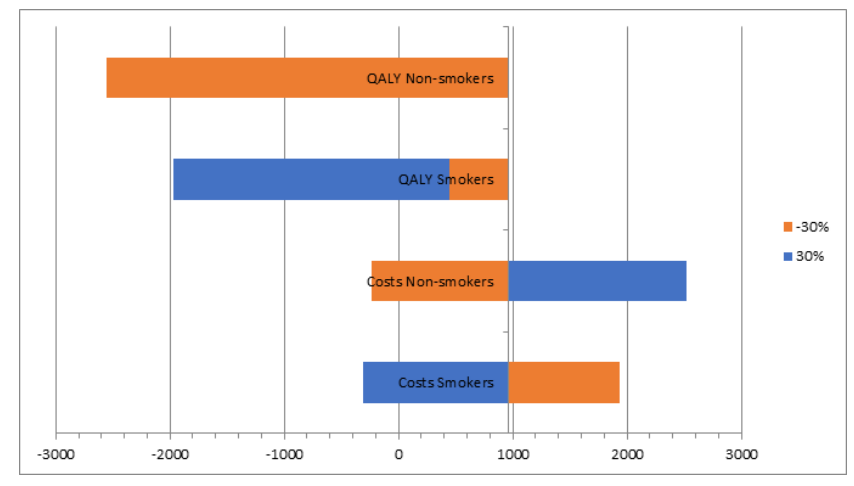

Figure 2. Tornado diagram with costs and QALYs varied $\pm 30 \%$ for smokers and non-smokers in the 426 patient cohort.

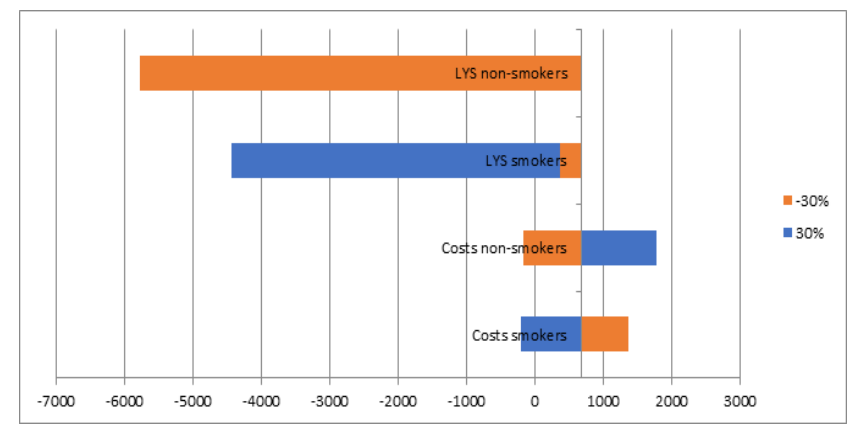

Figure 3. Tornado diagram with costs and LYSs varied $\pm 30 \%$ for smokers and non-smokers in the 426 patient cohort.

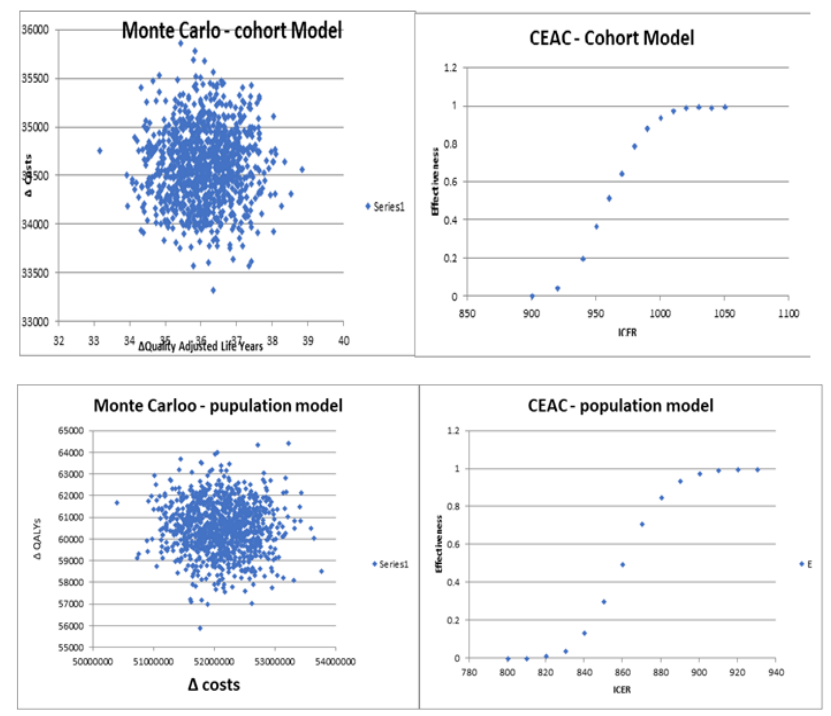

Figure 4. Monte Carlo distributions and cost-effectiveness acceptability curves for the cohort model and population model. 
Table 1. Initial patient distribution, Costs and Quality Adjusted Life Years (QALYs) per disease stage in the cohort.

\begin{tabular}{lllll}
\hline & GOLD A & GOLD B & GOLD C & 65 \\
\hline Initial dist. & 30 & 149 & 182 \\
\hline therapy cost & $438(349.43-523.56)$ & $591(505.43-676.52)$ & $773(687.44-858.56)$ & $790(704.44-875.56)$ \\
\hline Quality of life (QALY) & 0.85 & 0.81 & 0.76 \\
\hline
\end{tabular}

Table 2. Model results, costs and outcomes within the cohort of 426 patients.

\begin{tabular}{|c|c|c|c|c|c|}
\hline Disease stage & GOLD 1 & GOLD 2 & GOLD 3 & GOLD 4 & Dead \\
\hline Initial distr. & 30 & 149 & 65 & 182 & 0 \\
\hline Smokers cycle 10 & 17 & 98 & 66 & 15 & 230 \\
\hline \multirow[t]{2}{*}{ Non-smokers cycle 10} & 27 & 135 & 52 & 36 & 176 \\
\hline & Costs & QALY & CER & Mortality & LYS \\
\hline Smokers & 1520560.4 BGN & 1786.8 & 851.01 & $53.70 \%$ & 197 \\
\hline Non-smokers & 1866675.2 BGN & 2149.6 & 869.45 & $41.30 \%$ & 250 \\
\hline Difference $(\Delta)$ & $346114.8 \mathrm{BGN}$ & 362.8 & & & 53 \\
\hline Cost/result & & & $\mathrm{ICE}=954.1$ & & ICER $=6530.46$ \\
\hline
\end{tabular}

\section{Discussion}

This study is the first of its kind for Bulgaria and compares two hypothetical situations-one where all COPD patients are smokers and one where all are non-smokers. This way the model evaluates the benefit of smoking cessation on disease progression, as well as the associated burden, and the costeffectiveness of the current treatment options for COPD patients in the country. The strong points of the model are, that it is based in real-world data obtained from a representative cohort for the country-the prescribing information used revealed the character of prescribing practices nation-wide, the respective cost of therapy, and the percentage of patients within the GOLD classification. Combining this information with previously published studies estimating lung function decline and benefits of good disease control [17] allowed for the construction of a first for the country Markov model- one that evaluates future trends in chronic obstructive pulmonary disease, effectiveness of therapy plus life years saved, and impact of smoking status for both a representative cohort and the entire Bulgarian COPD population.

Table 3. Markov model distribution of patients at year 0 and year 10 for the entire Bulgarian population. *Note the significantly higher proportion of smokers $(310,465$ vs. 136,604) $39 \%$ of entire population $>40$ smoke.

\begin{tabular}{|c|c|c|c|c|c|}
\hline & GOLD 1 & GOLD 2 & GOLD 3 & GOLD 4 & Dead \\
\hline Smokers & 15635 & 109445 & 51372 & 134013 & 0 \\
\hline \multicolumn{6}{|l|}{ Initial Dist. } \\
\hline Smokers (Cycle 10) & 8896 & 69999 & 49634 & 11291 & 170634 \\
\hline Non-smokers & 10910 & 45535 & 18973 & 61187 & 0 \\
\hline \multicolumn{6}{|l|}{ Initial dist. } \\
\hline $\begin{array}{l}\text { Non-smokers cycle } \\
10\end{array}$ & 9870 & 41358 & 15311 & 11600 & 58464 \\
\hline
\end{tabular}

Table 4. Markov model results and outcomes for the ten-year period.

\begin{tabular}{|c|c|c|c|c|c|}
\hline & Costs & QALY & CER & Mortality & LYS \\
\hline Smokers & $1109121870.6 \mathrm{BGN}$ & 1283208 & 864.33 & $54.90 \%$ & 139861 \\
\hline Non-smokers & 588084255.7 BGN & 677581.3 & 867.91 & $42.70 \%$ & 78140 \\
\hline Difference $(\Delta)$ & $521037614.9 \mathrm{BGN}$ & 605626.7 & & & 61721 \\
\hline Cost/result & & & ICER $=860.32$ & & ICER=8441.82 \\
\hline
\end{tabular}

One limitation of our model is its static nature which focuses on COPD therapy in general within only 2 risk groups, not accounting for comorbidities. Therefore, costs remained stable and reflected only one dimension of the complex nature of treatment of this disease. Accordingly, even large variations of costs and effects in the sensitivity analysis $( \pm 30 \%)$ kept the incremental ratio well below the threshold of 3 times GDP per capita (treatment remained cost effective in the interval -2557.40 to +2516.29 for each QALY gained, and within -5768.58 to +1776.7 for each LYS gained). 
Other existing, dynamic models which predict the future of COPD therapy for a respective region are those of Rutten-van Molken and Martine Hoogedoorn for the healthcare system of the Netherlands, which was later extended to encompass also that of the UK and Scotland by MacLean [18,19]. The Hoogedorn model estimates the savings collected by implementing a smoking consultation service and concluded that "Minimal counseling cost saving and intensive counseling is less than $€ 15,000$ per QALY". The MacLeen study designed for England and Scotland concluded: "projected substantial year-on-year increases in the numbers of people with COPD over the period 2011-2030. These projections are similar to the Netherlands showing an increase, especially among older female patients. As a result of the increase in the prevalence, the models projected an increase in direct healthcare costs for the overall COPD population, and also an increase in the number of deaths from all causes among people with COPD."

Similar to our approach, Martine Hoogendoorn designed another model [20] which compared and validated existing COPD models, whereby all results were recalculated under the same hypothetical scenario and came to the following conclusions: "The authors receive as a result that the 5-year incremental cost-effectiveness ratios (ICERs) for the most comprehensive intervention was $€ 17,000$ /quality-adjusted lifeyear (QALY) for two models, €25,000 to €28,000/QALY for three models, and $€ 47,000 / \mathrm{QALY}$ for the remaining two models. They pointed out that the main factors leading to differences in the ICERs could mainly be explained by differences in input values for disease progression, exacerbation-related mortality, and all-cause mortality, with high input values resulting in low ICERs and vice versa. Lifetime results were mainly affected by the input values for mortality."

Four other models also focus mainly on the cost-effectiveness of smoking prevention treatments and programs with respect to lowering the burden of COPD [21-24] The cost-effectiveness of the explored alternatives differs in great extend from Can $\$ 17000$ (Sin) to Can $\$ 75000$ (Spenser).

The reason our study focused on smokers or non-smokers was because Bulgaria is among the countries with the highest number of active smokers. This has led experts to predict that the incidence of COPD for the country might increase two-fold to circa 900 thousand people by 2030, further emphasizing the need of smoking cessation programs. This seems consistent with WHO predictions, which put the disease as the second most common right after diabetes by 2030 . This prompted our interest in estimating the costs and outcomes for treatment of smokers and non-smokers and the corresponding costeffectiveness. We confirmed that the therapy is cost-effective within a large distribution (-2557.40 to +2516.29), while incremental ratio for the population is approximately $860 \mathrm{BGN}$ per QALY. Therefore, we suggest that emphasis should be given on educating the population and investing in smoking consultation and prevention schemes. Although this study presents a small aspect of a complex disease, it contributes to ongoing efforts of studying a developing region within the European Community of Nations.

\section{Conclusion}

Current COPD treatments for Bulgaria are effective in managing disease severity and slowing progression but are undercut by the large proportion of the population who smoke. The relatively high number of smokers with COPD account for nearly twice the cost of therapy with a lower quality of life. We confirmed non-smokers live longer and in generally better health. Smoking cessation campaigns could help lessen the health authority's economic burden.

\section{Acknowledgments}

The authors would like to thank the entire team of researchers in the COPD project for Bulgaria for the participation and data collection.

\section{References}

1. Murray CJL, Lopez AD. The Global Burden of Disease: a comprehensive assessment of mortality and disability from disease injuries and risk factors in 1990 and projected to 2020. Cambridge: Harvard University Press 1996.

2. Bousqet J, Khaltaev N. Global surveillance, prevention and control of chronic respiratory diseases. A comprehensive approach. global alliance against chronic respiratory disease. Geneva WHO Press 2007.

3. WHO. Global surveillance, prevention and control of chronic respiratory diseases: a comprehensive approach. Available from: http: //www.who.int/respiratory/ publications/en/

4. Perret JL, Dharmage SC, Matheson MC, Johns DP, Gurrin LC, Burgess JA, Marrone J, Markos J, Morrison S, Feather I, Thomas PS, McDonald CF, Giles GG, Hopper JL, Wood-Baker R, Abramson MJ, Walters EH. The interplay between the effects of lifetime asthma, smoking, and atopy on fixed airflow obstruction in middle age. Am J Respir Crit Care Med 2013; 187: 42-48.

5. Quach A, Giovannelli J, Cherot-Kornobis N. Prevalence and underdiagnosis of airway obstruction, among middleaged adults in northern France: the ELISABETH study 2011-2013. Respir Med 2015: 109: 1553-1561.

6. Halbert RJ, Natoli JL, Gano A, Badamgarav E, Buist AS, Mannino DM. Global burden of COPD: systematic review and meta-analysis. Eur Respir J 2006; 28: 523-532.

7. Lamprecht B, McBurnie MA, Vollmer WM, Gudmundsson G, Welte T, Nizankowska-Mogilnicka E, Studnicka M, Bateman E, Anto JM, Burney P, Mannino DM, Buist SA, BOLD Collaborative Research Group. COPD in never smokers: results from the populationbased burden of obstructive lung disease study. Chest 2011; 139: 752-763.

8. Terzikhan N, Verhamme KM, Hofman A, Stricker BH, Brusselle GG, Lahousse L. Prevalence and incidence of 
COPD in smokers and non-smokers: the Rotterdam Study. Eur J Epidemiol 2016; 31: 785-792.

9. GOLD-Pocket guide to copd diagnosis, management, and prevention. A Guide for Health Care Professionals 2015.

10. Feenstra TL, van Genugten ML, Hoogenveen RT. The impact of aging and smoking on the future burden of chronic obstructive pulmonary disease: a model analysis in the Netherlands. Am J respir Crit Care Med 2001; 164: 590-596.

11. Atsou K, Chouaid C and Hejblum G. Simulation-based estimates of effectiveness and cost-effectiveness of smoking cessation in patients with chronic obstructive pulmonary disease. PLos One 2011; 6: 24870.

12. www.ncpr.bg

13. Chandra K, Blackhouse G, McCurdy BR, Bornstein M, Campbell K, Costa V, Franek J, Kaulback K, Levin L, Sehatzadeh S, Sikich N, Thabane M, Goeree R. Costeffectiveness of interventions for chronic obstructive pulmonary disease (COPD) using an Ontario policy model. Ont Health Technol Assess Ser 2012; 12: 1-61.

14. Petra M, Reiner L, Rolf H. A lifetime Markov model for the economic evaluation of chronic obstructive pulmonary disease. Pharmacoeconomics 2012: 30: 825-840.

15. Chandra K, Blackhouse G, McCurdy BR, Bornstein M, Campbell K, Costa V, Franek J, Kaulback K, Levin L, Sehatzadeh S, Sikich N, Thabane M, Goeree R. Costeffectiveness of interventions for chronic obstructive pulmonary disease (COPD) using an Ontario policy model. Ont Health Technol Assess Ser 2012; 12: 1-61.

16. Paul DS, John EC, Lance AW. Smoking cessation and lung function in mild-to-moderate chronic obstructive pulmonary disease. The Lung Health Study. Am J Respir Care Med 2000; 163: 381-390.

17. Chandra K, Blackhouse G, McCurdy BR, Bornstein M, Campbell K, Costa V, Franek J, Kaulback K, Levin L, Sehatzadeh S, Sikich N, Thabane M, Goeree R. Costeffectiveness of interventions for chronic obstructive pulmonary disease (COPD) using an Ontario policy model. Ont Health Technol Assess Ser 2012; 12: 1-61.

18. Hoogendoorn M, Rutten-van Mölken MP, Hoogenveen RT, van Genugten ML, Buist AS, Wouters EF, Feenstra
TL. A dynamic population model of disease progression in COPD. Eur Respir J 2005; 26: 223-233.

19. McLean S, Hoogendoorn M, Hoogenveen RT, Feenstra TL, Wild S, Simpson CR, Mölken MR, Sheikh A. Projecting the COPD population and costs in England and Scotland: 2011 to 2030. Sci Rep 2016; 6: 31893.

20. Martine H, Talitha LF, Yumi A, Sixten B, Ryan NH, Sven AJ, Yevgeniy S, Margarethe W, Andrew HB, Adam L, Sean DS, Maureen PMH, Rutten-van M. Costeffectiveness models for chronic obstructive pulmonary disease: cross-model comparison of hypothetical treatment scenarios. Value Health 2014; 17: 525-536.

21. Sin DD, Golmohammadi K, Jacobs P. Cost-effectiveness of inhaled corticosteroids for chronic obstructive pulmonary disease according to disease severity. Am J Med 2004; 116: 325-331.

22. Borg S, Ericsson A, Wedzicha J, Gulsvik A, Lundback B, Donaldson GC, Sullivan SD. A computer simulation model of the natural history and economic impact of chronic obstructive pulmonary disease. Value Health 2004; 7: 153-167.

23. Spencer M, Briggs AH, Grossman RF, Rance L. Development of an economic model to assess the cost effectiveness of treatment interventions for chronic obstructive pulmonary disease. Pharmacoeconomics 2005; 23: 619-637.

24. Oostenbrink JB, Rutten-van Molken MP, Monz BU, FitzGerald JM. Probabilistic Markov model to assess the cost-effectiveness of bronchodilator therapy in COPD patients in different countries. Value Health 2005; 8: $32-46$.

\section{*Correspondence to}

Konstantin Tachkov

Medicinski Universitet Sofia

Sofia

Bulgaria 